EPJ Web of Conferences 82, 01016 (2015)

DOI: $10.1051 /$ epjconf/20158201016

(C) Owned by the authors, published by EDP Sciences, 2015

\title{
The crystallization processes in the aluminum particles production technology
}

\author{
Vladimir Arkhipov ${ }^{1,2}$, Sergey Bondarchuk ${ }^{1,2}$, Victor Goldin ${ }^{1}$ and Irina Zharova ${ }^{1, a}$ \\ ${ }^{1}$ Tomsk State University, Scientific Research Institute of Applied Mathematics and Mechanics, Tomsk, \\ Russia \\ ${ }^{2}$ Institute for Problems of Chemical \& Energetic Technologies of the Siberian Branch of the Russian \\ Academy of Sciences, Biysk, Russia
}

\begin{abstract}
The physical and mathematical model of the crystallization process of liquid aluminum particles in the spray-jet of the ejection-type atomizer was proposed. The results of mathematical modeling of two-phase flow in the spray-jet and the crystallization process of fluid particles are given. The influence of the particle size, of the flow rate and the stagnation temperature gas in the ranges of industrial technology implemented for the production of powders aluminum of brands ASD, on the crystallization characteristics were investigated. The approximations of the characteristics of the crystallization process depending on the size of the aluminum particles on the basis of two approaches to the mathematical description of the process of crystallization of aluminum particles were obtained. The results allow to optimize the process parameters of ejection-type atomizer to produce aluminum particles with given morphology.
\end{abstract}

\section{Introduction}

Optimization of technologies for the production of highly dispersed metal powders is important in view of a sustainable trend for extending the application of powder materials. In particular, powders of aluminum are used as materials of high-energy combustible components [1-3]. Gas-dynamic dispersion jet of molten metal by ejection-type atomizer is the most common industrial technology of a large number of ways to obtain highly dispersed metal powders [4-7]. Production of metal powder by ejection-type atomizer include spray melt to form the liquid polydispersed particles, motion and crystallization of the liquid particles in the flow of the spray gas. Existing technology of industrial production of aluminum powders brands ASD [8] provides content of highly dispersed fraction $\sim 18 \%$ (particle diameter less than $10 \mu$ ) in the spray ejection-type atomizer. Improvement of the existing technology spraying of the melt aluminum by compressed gas is needed to increasing the yield of the particles of highly dispersed fraction and for increasing of homogeneity of the dispersed particles composition of the final product. A number of scientific and practical problems arise in this case.

\footnotetext{
${ }^{a}$ Corresponding author: zharova@niipmm.tsu.ru
}

This is an Open Access article distributed under the terms of the Creative Commons Attribution License 4.0, which permits unrestricted use, distribution, and reproduction in any medium, provided the original work is properly cited. 


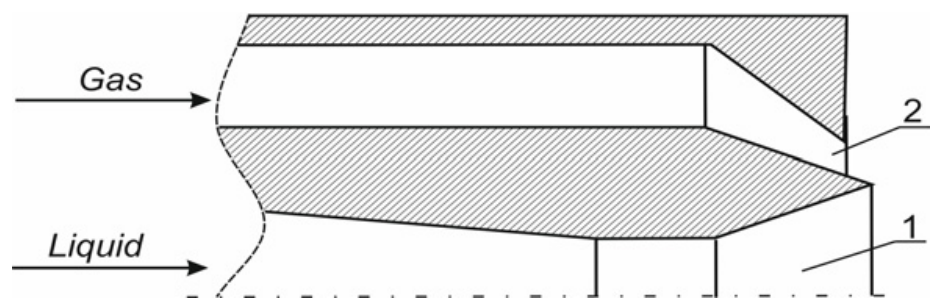

Figure 1. The scheme of the block of the ejection-type atomizer. 1 - A melt flow channel; 2 - A atomizing gas flow channel.

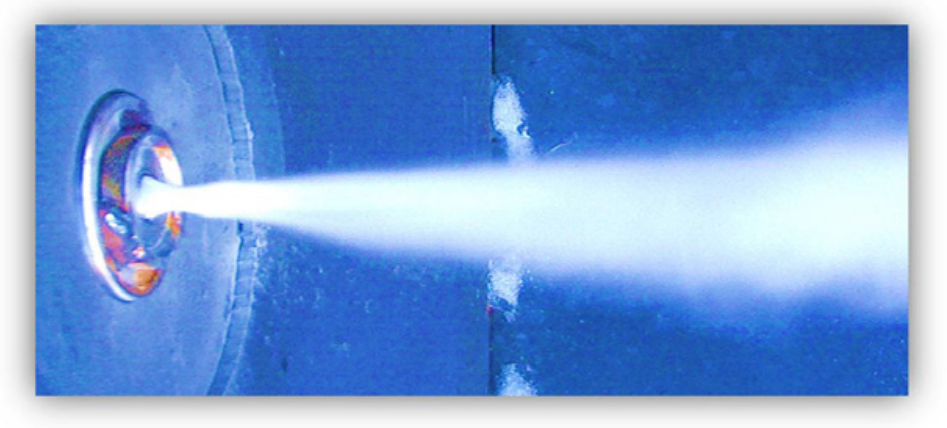

Figure 2. The spray-jet of the ejection-type atomizer.

Investigation of the influence of the time and the dynamics of the phase transition on the aluminum particles morphology refers to the number of such problems.

The results of mathematical modeling of two-phase flow in the spray jet and of the crystallization process of liquid aluminum particles are given in this paper.

The scheme of the block of the ejection-type atomizer and photo of the spray-jet are shown in Figs. 1, 2.

The molten metal is fed through the central channel 1 and flows out of the outlet section as an annular jet. The flow of hot gas (nitrogen) is supplied through the annular channel 2. Estimates of the liquid jet stability indicate, that its decomposition occurs with the formation of polydisperse aerosol in the immediate vicinity of the outlet to working chamber. that its decay occurs with the formation of polydisperse aerosol in the immediate vicinity of the outlet to working chamber.

\section{Modeling of the liquid particles aluminum crystallization in the spray-jet of the ejection-type atomizer}

The physical model is based on the following assumptions. Fluxes of mass, momentum and energy of the gas comes from the annular injector nozzle for the given stagnation gas parameters with critical parameters corresponding to the value of the Mach number $M=1$. The flow of gas occurs in a mode of insufficient expansion, since the static pressure in the jet at the nozzle outlet is more than in the environment, $p_{g}>p_{e}$. The process of heat transfer when the particles move in the gas jet is carried out by convection predominantly, because the Biot number is $\mathrm{Bi} \ll 0.1$. Liquid particles are spherical. Particle temperature is uniform in volume and depends only on time. 
In modeling took into account that the diameters of the liquid particles in the spray-jet are in the range $d_{p}=(2 \div 50) 1$ under the current technology for production of industrial aluminum powders brands ASD.

The temperature regime of the particles in the collecting chamber was considered as a three- stages: 1) it's cooling to a temperature of melting, 2) it's crystallization, 3) it's cooling to the temperature environment in the chamber. Total time $t_{\Sigma}$, required to cooling to complete curing of the liquid particle, was defined as the sum of the cooling time of the particle to the crystallization temperature $\left(t_{1}\right)$ and crystallization time $\left(t_{2}\right)$. Relations for the approximate estimation of the time $t_{1}$ and $t_{2}$ were obtained from the heat balance equation in the assumption of a constant gas temperature:

$$
\begin{gathered}
t_{1}=\frac{c_{k} \rho_{k} d_{p}^{2}}{6 N u \lambda_{g}} \ln \frac{T_{m}-T_{g}}{T_{k r}-T_{g}}, \\
t_{2}=\frac{q_{k r} \rho_{k} d_{p}^{2}}{6 \mathrm{Nu}\left(T_{k r}-T_{g}\right) \lambda_{k}},
\end{gathered}
$$

where $\rho_{k}, c_{k}, \lambda_{k}$ are the density, the specific heat capacity and coefficient of the thermal conductivity of particle; $\lambda_{g}$ is coefficient of the gas thermal conductivity; $T_{g}$ is the gas temperature; $T_{m}, T_{k r}$ are the initial temperature and the crystallization temperature of the particle; $q_{k r}$ is heat of the phase transition (specific); Nu is the Nusselt number.

Heat transfer between the particle and the carrier gas jet is determined by Newton's law in conditions of convection:

$$
q=\alpha\left(T_{k}-T_{g}\right)
$$

where $\alpha$ is the heat transfer coefficient; $T_{k}$ is the temperature of particle.

The heat transfer coefficient for spherical particles in two-phase flow for Reynolds numbers in the range of $\operatorname{Re} \geq\left(150 \div 3 \cdot 10^{4}\right)$ determined by the formula [9]:

$$
\mathrm{Nu}=0.62 \operatorname{Re}^{0.5} \text {. }
$$

The investigated process was modeled under the assumptions made using two approaches. Solution of the problem of the outflow of the gas jet to the working chamber was considered in the first approach in the formulation based on the inviscid Euler equations in the perfect gas:

$$
\begin{aligned}
& \frac{\partial}{\partial x}\left(\rho_{g} u_{g}\right)+\frac{1}{r} \frac{\partial}{\partial r}\left(\rho_{g} v_{g} r\right)=0 \\
& \frac{\partial}{\partial x}\left(\rho_{g} u_{g}^{2}\right)+\frac{1}{r} \frac{\partial}{\partial r}\left(\rho_{g} u_{g} v_{g} r\right)=-\frac{\partial p_{g}}{\partial x} \\
& \frac{\partial}{\partial x}\left(\rho_{g} u_{g} v_{g}\right)+\frac{1}{r} \frac{\partial}{\partial r}\left(\rho_{g} v_{g} r^{2}\right)-\frac{\rho_{g} u_{g}^{2}}{r}=-\frac{\partial p_{g}}{\partial r} ; \\
& \frac{\partial}{\partial x}\left[u_{g}\left(\rho_{g} E+p_{g}\right)\right]+\frac{1}{r} \frac{\partial}{\partial r}\left[v_{g}\left(\rho_{g} E+p_{g}\right) r\right]=0,
\end{aligned}
$$

where $x, r$ are the axial and radial coordinates; $t$ is the time; $\rho$ is the density; $u_{g}, v_{g}$ are the axial and radial velocity components; $p_{g}$ is the pressure; $h$ is the static enthalpy (specific),

$$
h=\frac{\gamma}{\gamma-1} \frac{p_{g}}{\rho_{g}}
$$

where $\gamma$ is the adiabatic index; $E$ is the total energy per unit mass,

$$
E=h-\frac{p_{g}}{\rho_{g}}+\frac{1}{2}\left(u_{g}^{2}+v_{g}^{2}\right) .
$$




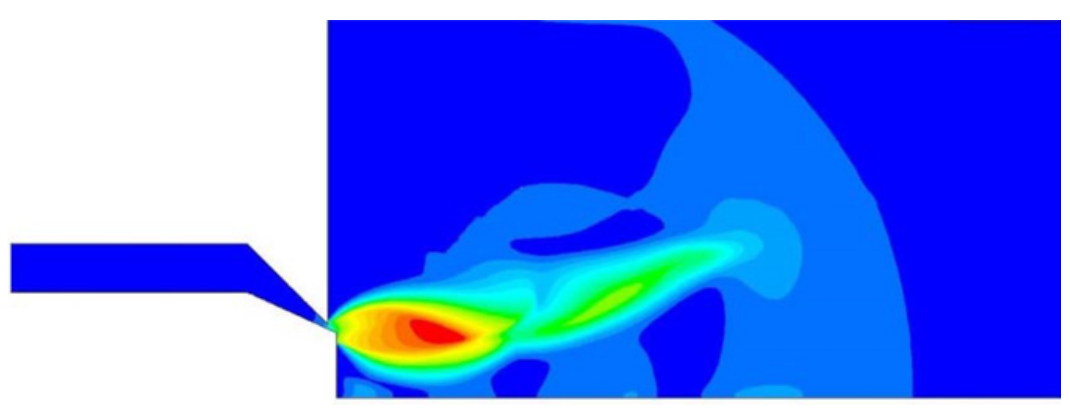

Figure 3. Mach number in the flow region of gas.

Stagnation gas parameters and Mach number were given on the entrance to the channel 2 (see Fig. 2). Symmetry conditions were set on the jet axis, the conditions of impermeability were set on solid walls, the environment pressure $p_{e}$ was set at the outer edge.

Dynamics of particles in the gas phase was calculated under the trajectory approach.

Influence of particles dynamics on the gas parameters was taken into account in the second approach. Euler equations in the one-dimensional approximation were solved simultaneously with the equations for multicomponent "gas" of particles $[10,11]$. Change in cross-sectional jet diameter was taken into account before the first Mach disc, further the diameter of the cross section of the jet was considered as constant. The mutual influence of phases was taken into account with the help of relations describing the interphase exchange of momentum and energy.

\section{Analysis of the numerical results}

Influence of parameters of spray gas (nitrogen) on the main characteristics (crystallization time and the distance from the exit section) crystallization of aluminum droplets of different sizes was investigated in numerical experiments.

Parametric calculations were performed for fixed values of the width of the annular channel $H=0.54 \mathrm{~mm}$ and of the area of the annular exit section nozzle $S_{c}=27 \mathrm{~mm}^{2}$. The following parameters of the spray gas were varied, the volumetric consumption $Q=(600 \div 900) \mathrm{m}^{3} / \mathrm{h}$, the stagnation temperature $T_{0}=(500 \div 600)^{\circ} \mathrm{C}$. The consumption of the molten metal amounted to $150 \mathrm{~kg} / \mathrm{h}$, the film thickness of the melt at the outlet of the chamber amounted to $70 \mathrm{~mm}$, velocity of the film amounted to $5 \mathrm{~m} / \mathrm{s}$.

The task of (1) was solved using the software package ANSYS Fluent version 13.0 in axisymmetric formulation (first approach). Unsteady explicit method Rowe with the first order of approximation in the space variables and the time was used as a method of solution. The results of one of the calculations are shown in Figs. 3, 4 for the following values of the flow parameters at the channel inlet: the stagnation temperature $T_{0}=773 \mathrm{~K}$, pressure $p_{0}=6.08 \cdot 106 \mathrm{~Pa}$, the Mach number $\mathrm{M}=0.055$.

The results of numerical research, obtained on the basis of the second approach with the particle diameter $d_{p}=25 \mu$ at the stagnation temperature $T_{0}=575 \mathrm{C}$ and for various flow velocities of the spray gas, are shown in Figs. 5-8.

The main characteristics of the process such as the time $t_{0}$ and the coordinate $x_{0}$ along the jet axis, corresponding to the beginning of crystallization, $t_{\Sigma}$ duration of the process and length of the section $S$ of crystallization, were calculated when analyzing the influence of gas consumption $Q$ and the stagnation temperature $T_{0}$ on the crystallization of aluminum particles. The results of calculations were approximated using power dependences of the form:

$$
f\left(d_{p}\right)=a d_{p}^{b},
$$




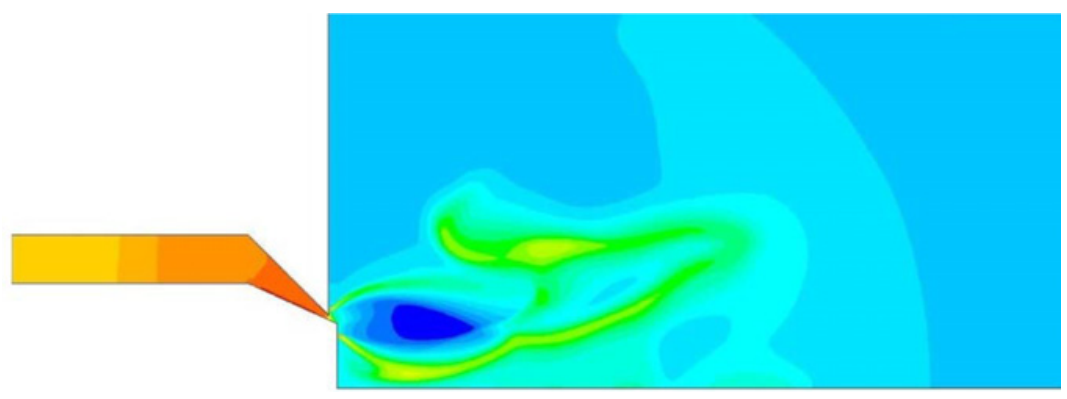

Figure 4. The temperature distribution.

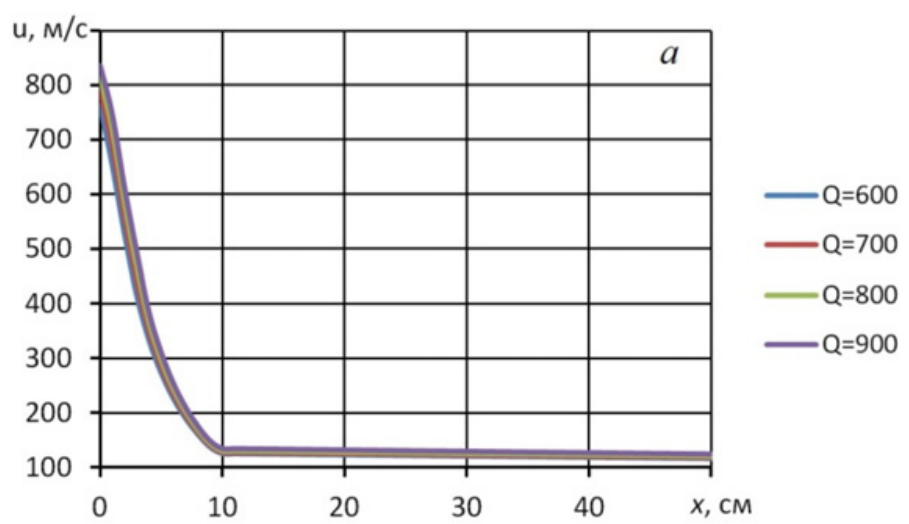

Figure 5. The change of the gas velocity along a jet depending on the gas consumption.

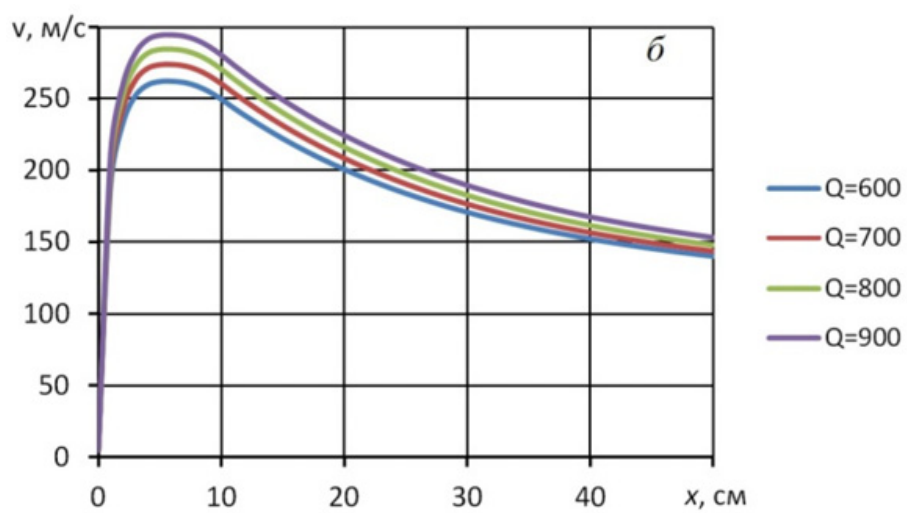

Figure 6. The change of the particles velocity along a jet depending on the gas consumption.

where $f\left(d_{p}\right)$ is one of the four characteristics of crystallization; $\left[d_{p}\right]=\mu$, the particles diameters are in the range $d_{p}=(2 \div 50) 1 ; a, b$ are the approximation constants. The resulting constants of approximation when the gas consumption $Q=(600 \div 900) \mathrm{m}^{3} / \mathrm{h}$ are shown in Table 1 . 


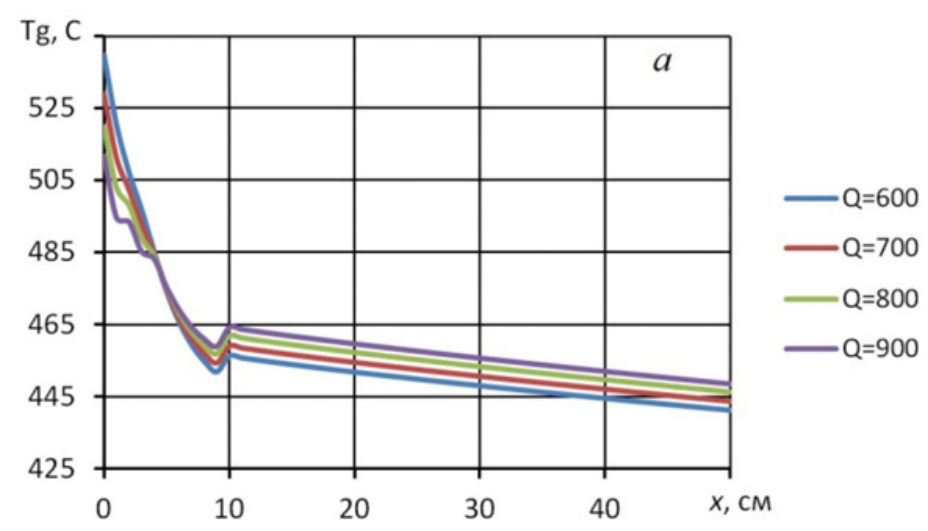

Figure 7. The change of the gas temperature along a jet depending on the gas consumption.

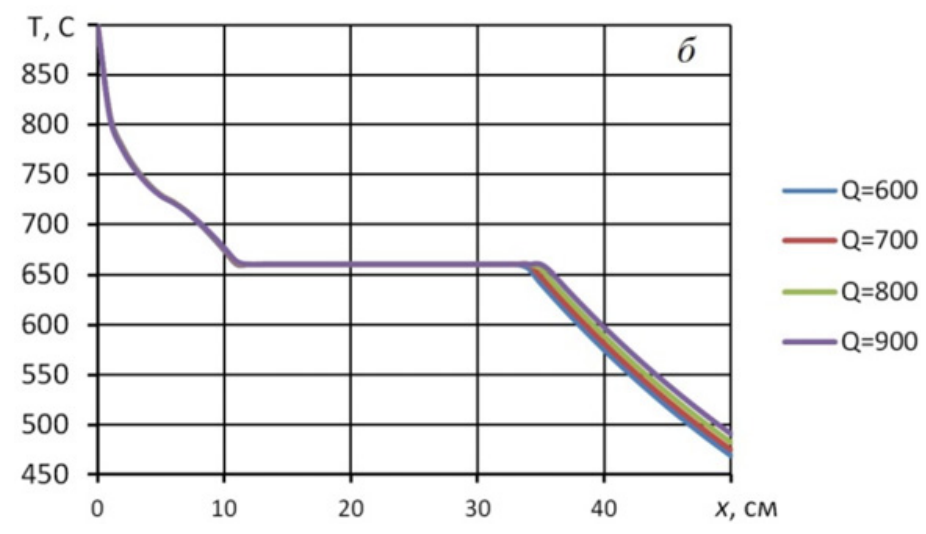

Figure 8. The change of the particles temperature along a jet depending on gas consumption.

Table 1. The constants of approximation for the crystallization characteristics.

\begin{tabular}{|c|c|c|c|c|}
\hline$Q, \mathrm{~m}^{3} / \mathrm{h}$ & $t_{0}, \mathrm{~ms}$ & $t_{\Sigma}, \mathrm{ms}$ & $x_{0}, \mathrm{~cm}$ & $S, \mathrm{~cm}$ \\
\hline 600 & $0.17 \cdot 10^{-2} d_{p}^{1.790}$ & $0.34 \cdot 10^{-2} d_{p}^{1.836}$ & $0.36 \cdot 10^{-1} d_{p}^{1.741}$ & $0.227 d_{p}^{1.465}$ \\
\hline 700 & $0.16 \cdot 10^{-2} d_{p}^{1.784}$ & $0.33 \cdot 10^{-2} d_{p}^{1.841}$ & $0.36 \cdot 10^{-1} d_{p}^{1.743}$ & $0.231 d_{p}^{1.462}$ \\
\hline 800 & $0.16 \cdot 10^{-2} d_{p}^{1.780}$ & $0.32 \cdot 10^{-2} d_{p}^{1.848}$ & $0.36 \cdot 10^{-1} d_{p}^{1.747}$ & $0.233 d_{p}^{1.462}$ \\
\hline 900 & $0.16 \cdot 10^{-2} d_{p}^{1.777}$ & $0.32 \cdot 10^{-2} d_{p}^{1.844}$ & $0.35 \cdot 10^{-1} d_{p}^{1.753}$ & $0.235 d_{p}^{1.465}$ \\
\hline
\end{tabular}

\section{Conclusion}

Analysis of the results of numerical studies of crystallization of aluminum particles in the spray-jet of the ejection-type atomizer showed:

- the flow rate and the stagnation temperature of the spray-jet gas in the ranges, which realized in the industrial technology for producing aluminum powders of brands ASD, have practically no influence on the characteristics of crystallization - the crystallization process mainly depends on the particle size;

- begin time of crystallization increases from 6 ls to $1.9 \mathrm{~ms}$ with increasing the initial particle size of 2 to $50 \mu$; 
- the initial coordinate of crystallization is increased in the range $(0.12 \div 33) \mathrm{cm}$ with increasing the initial particle size of 2 to $50 \mu$;

- the crystallization duration increases in the range $(0.01 \div 4.5)$ ms with increasing the initial particle size of 2 to $50 \mu$;

- the crystallization length increases in the range $(0.6 \div 7) \mathrm{cm}$ with increasing the initial particle size of 2 to $50 \mu$;

- these parameters allow you to completely define the morphology of the particles formed of aluminum.

The proposed physical-mathematical model of the process of crystallization of liquid aluminum particles in the spray-jet of the ejection-type atomizer and obtained crystallization process characteristics approximations (duration of the crystallization process, the time and the coordinate along the jet axis, corresponding to the beginning of crystallization, the length of crystallization section) of the particles size of the aluminum allow to optimize the process parameters by the ejection-type atomizer for producing aluminum particles with given morphology.

${ }^{1}$ This work was financially supported by the Ministry of Education and Science of the Russian Federation within the framework of state job No. 2014/223 (project No. 1567) and of state job No. 9.1024.2014/K.

\section{References}

[1] L. Meda, G. Marra, L. Galfetti, F. Severini, and L. De Luka, Mater. Sci. and Eng., 27, 5 (2007)

[2] J.R. Luman, B. Wehrman, K.K. Kuo, R.A. Yetter, N.M. Masoud, T.G. Manning, L.E. Harris, and H.A. Bruck, Proceedings of the Combustion Institute, 31 (2007)

[3] C. Rossi, A. Estéve, and P. Vashishta, Journal Phys. Chem. Solids, 71, 2 (2010)

[4] A.M. Sizov, Gas dynamics and heat transfer of the gas jets in metallurgical processes (Moscow, Metallurgiya, 1987)

[5] A.M. Sizov, Dispersing the melts by the supersonic jets gas (Moscow, Metallurgiya, 1991)

[6] C.N.R. Rao, A. Müller, A.K. Cheetham, Nanomaterials Chemistry: Recent Developments and New Directions (Weinheim: Viley-VCH Verlag, 2007)

[7] Y. Xiao , L.Qi, X. Zeng, Y. Chao, and F. Yang, Journal of Mechanical Engineering, 47, 15 (2011)

[8] Aluminum powder highly dispersed ASD-1, ASD-4, ASD-6: Technical conditions 48-5-28-87 (LLC “SUAL-PM", Shelekhov, 1987)

[9] O.S. Nichiporenko, Yu.I. Naida, A.B. Medvedovsky, Atomizing of metal powders (Kiev, Naukova Dumka, 1980)

[10] I. Vasenin, V. Arkhipov, V. Butov, A. Glazunov, V. Trofimov, Gas Dynamics of Binary Flows in Nozzles(Tomsk, TSU Publishing House, 1986)

[11] G. Abramovich, T.A. Girshovich, S.Yu. Krasheninnikov, A.N. Sekundov, I.P. Smirnova, Theory of Turbulent Jets (Moscow, Nauka, 1984) 